\title{
Automated Calibration Of Greenhouse Energy Model Using Hybrid Evolutionary Programming (EP)-Energy Plus
}

\author{
NY Dahlan, S Z Sakimin, M. Faizwan, N. Ajmain, AA Aris \\ Faculty of Electrical Engineering, Universiti Teknologi MARA, 40450 Shah Alam, Selangor, Malaysia
}

\begin{tabular}{l}
\hline Article Info \\
\hline Article history: \\
Received May 4, 2018 \\
Revised Jul 5, 2018 \\
Accepted Jul 19, 2018 \\
\hline
\end{tabular}

\section{Keywords:}

Calibrated energy simulation Enery plus

Evolutionary programming

Greenhouse system

\begin{abstract}
This paper presents an optimization approach of calibrating a tomato greenhouse energy model using hybrid Evolutionary Programming (EP)EnergyPlus. The proposed methodology applies automated simulation-based approach by coupling Matlab and EnergyPlus to perform building energy simulation and obtain the best variables configuration with minimal error between the simulated and measured energy of the greenhouse. The proposed method is tested using a tomato greenhouse system located in Universiti Putra Malaysia (UPM). The greenhouse envelope is built using $0.15 \mathrm{~mm}$ thick Transparency Plastic Film. Meanwhile, the electrical loads in the greenhouse consists of 6 exhaust fans, 2 axial fans, 5 fluorescent lamps and 1 irrigation pump. An Evolutionary Programming (EP) algorithm is chosen and programmed in Matlab to find the best configurations for optimum calibration of the greenhouse energy model. Three variables were chosen to find the best configuration which are the operating hours of Exhaust Fan, Axial Fan and Water Pump. The EP optimization algorithm in Matlab is coupled with building energy simulator, EnergyPlus using BCVTB as the coupling tool. Result shows that the EnergyPlus-EP model can provide NMBE and CV(RMSE) within the range recommended by the IPMVP protocol. The proposed method is not only requiring less computation time but also effective in searching for the best variables configuration with minimal error.
\end{abstract}

Copyright $\odot 2018$ Institute of Advanced Engineering and Science. All rights reserved.

\section{Corresponding Author:}

NY Dahlan

Faculty of Electrical Engineering,

Universiti Teknologi MARA,

40450 Shah Alam, Selangor, Malaysia.

Email: nofriyenita012@ppinang.uitm.edu.my

\section{INTRODUCTION}

Automated calibration is a process that coupling the simulators to find the best variables configuration to an energy problem from available alternative configurations with desired objective function. Building simulation may involve a lot of parameters and variables including climate condition, HVAC, form and structure, operating schedule and electric equipment. Considering that, building involves non-linear interaction and complex function beyond human calculation capabilities. On the other hand, a great advancement in computational science and mathematical has been considered a bless in aiding the modelling, design, simulation and analysis. Energy Plus is an example of whole building simulator, which developed and funded by U.S Department of Energy (DOE) and has been widely employed to model both energy consumption and water use in a building. Currently there are 153 software listed out by DOE [1], including database, spreadsheets, components and system analysis that can be used to simulate building energy. However, most of the software listed can only be used for manual calibration method whereas users are expected to have a full complete and correct data in obtaining a precise baseline energy. This hypothetically, is time consuming and wasting processing power. 
Coupling software between Building Energy Simulator (BES) and algorithmic optimization engine has been found since late 2000s. There are a few built-in ready to be used coupling software for optimization such as ME+ [2]-[3], JEPlus [4] and GenOpt [5]. Despite having coupled together, most of the software are only limited to one or certain optimization algorithm. Building Control Virtual Test Bed (BCVTB) is a coupling software that allows users to couple different simulation program and/or with physical hardware itself for co-simulation. BCVTB is based on Ptolemy II software environment for an easier modelling. There is few simulation software that can be coupled together such as TRANSYS [6], Radiance, ESP-r and others including algorithmic optimization engine such as Matlab. The advancement in computer technologies has made the application of numerical optimization at ease since 1980s. Since then, BES have been modelled in term of mathematical/empirical Equations which obtained through rigorous energy simulation.

Evolutionary Algorithms (EAs) are new kinds of modern optimization algorithms that inspired by principle of nature evolution. EAs have some advantages over the traditional optimization algorithms and are of the great importance and have a wide range of applications. The traditional optimization algorithms usually have strict limitation on the functions such as their differentiability; however, EAs do not require the differentiability of the functions and have parallel property. Therefore, they are often used to solve some complex, large scale, nonlinear and non-differentiable optimization problems. There are varieties of optimization algorithm that has been developed such as Artificial Bee Colony (ABC) [7], Genetic Algorithms (GA) [8], particles warm optimisation (PSO) [9] and others which has its own specification and characteristic. Furthermore, a combination of one or few algorithms as a hybrid algorithm also has been introduced to improve the previous version in term of speed and data processing. EAs are search and optimization techniques based on the principal of natural evolution. There are four main streams in evolutionary algorithms namely Genetic Algorithms (GA), Genetic Programming (GP), Evolution Strategies (ES) and Evolutionary Programming (EP).

This paper presents an automated calibration of simulated baseline energy for a greenhouse system using Evolutionary Programming (EP) at minimum error between simulated and measured energy use. An optimization-based simulation is chosen and carried out by coupling the optimization algorithm engine, Matlab and Energy Plus with EP to determine baseline energy at minimal error. BCVTB is used to couple the Energy-plus with Matlab using Ptolemy II environment. A single objective function is defined and set in the EP to minimized error between simulated and measured energy from the greenhouse. Furthermore, three variables are randomized for initialization process to search for a desired group of population such as the operating hours of axial fan, exhaust fan and irrigation pump. The greenhouse building located in Universiti Putra Malaysia (UPM) campus is used as a case study.

\section{RESEARCH METHOD}

The methodology in this study comprises of six stages i.e. 1) project overall framework, 2) greenhouse modelling, 3) EP algorithm development, 4) building energy simulator 5) calibration process and 6) building energy evaluation.

\subsection{Project Overall Framework}

The overall project framework starts with modelling the greenhouse system using OpenStudio SketchUp. The energy simulation is carried out by using EnergyPlus. The EnergyPlus is used to build the greenhouse model by key-in the building data such as building parameters, HVAC setup, electrical equipment and etc. in the software. Meanwhile, Evolutionary Programming (EP) programed in the Matlab is used to optimize the calibration process in getting the minimum error. EP comprises of four different phases i.e. 1) initialization, 2) mutation and evaluation, 3) combination, selection and new generation and 4) convergence test.

To build an accurate greenhouse energy model, the model must pass a calibration process. This is performed by ensuring the difference between simulated and measured energy data is within a specified error by IPMVP protocol. The calibration involves an iterative process to find the best variables configuration in the greenhouse model to minimize error between simulated and measured energy use of the greenhouse. The variables are the operating hours of exhaust fan, axial fan and water pump for irrigation system. Building Control Virtual Test Bed (BCVTB) is used as a middle tool for the Matlab and EnergyPlus. The calibrated model is then used to estimate energy savings from several retrofit project.

\subsection{Modelling}

The greenhouse model is setup in such that the energy consumption will represent the actual greenhouse. With the available of Computer Aided Design (CAD) software and Graphical User Interface (GUI), the greenhouse model can be easily drawn in 3D and the thermal zone setting can be easily 
manipulated to get desired results. The building modelled in this paper is a Tomato Greenhouse located in Universiti Putra Malaysia (UPM) with total area of $100 \times 20 \mathrm{~m}^{2}$ (Figure 1). Figure 2 shows an adaptation of building model in 3D using Open-studio Sketch-up. The model has a height of 14 feet from ceiling to bottom, 29 feet wide and 100 feet length. Meanwhile, the electrical loads in the greenhouse includes 6 exhaust fans, 2 axial fans and 1 irrigation pump.
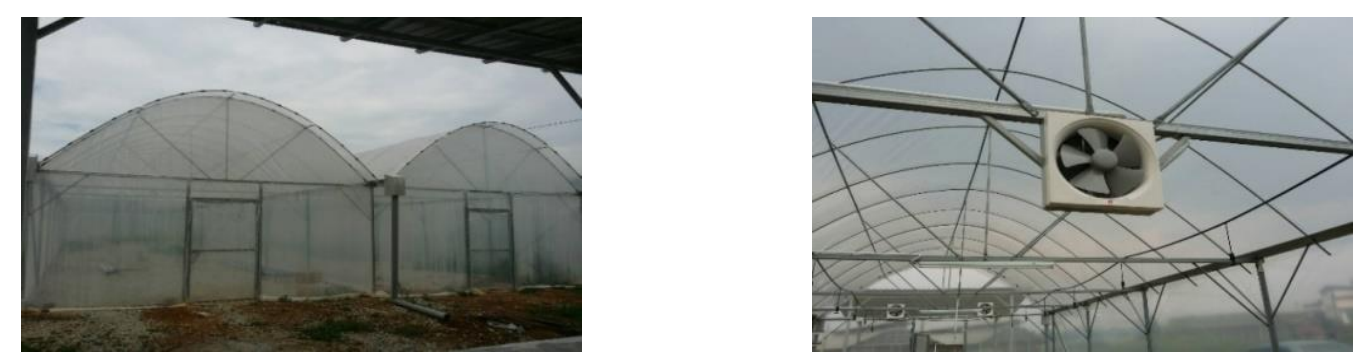

Figure 1. Greenhouse system located in UPM
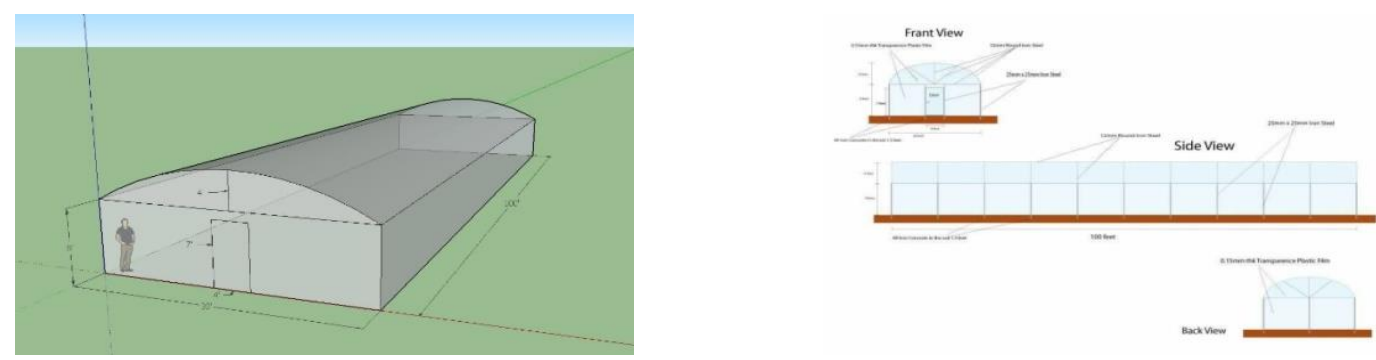

Figure 2. The schematic views of the building model

\subsection{Evolutionary Programming (EP)}

In this paper, the effectiveness of EP to solve baseline energy problem is explored. EP is a population-based generation and test approach, in which mutations are the search operator to generate new solutions. EP in effect has similar feature in stochastic selection but emphasizing more on the mutation and its variant operator. Figure 3 (a) shows a typical flow process in an EP.

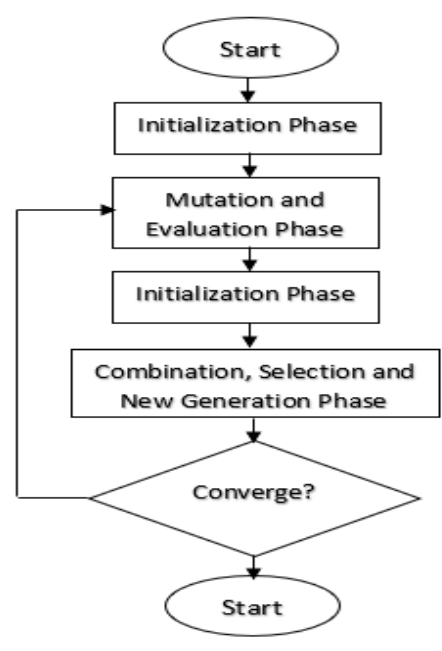

(a)

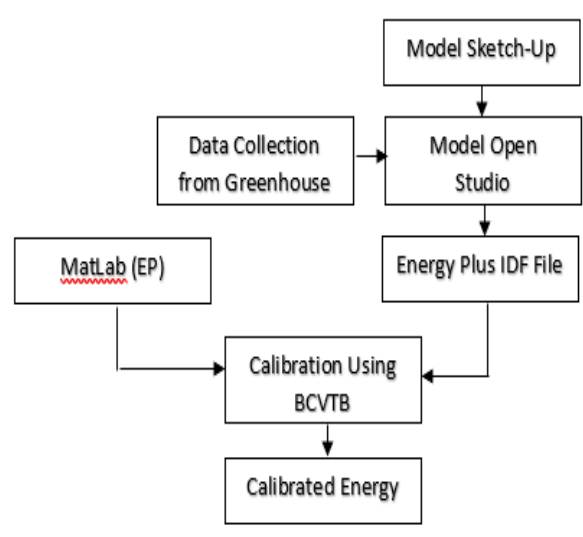

(b)

Figure 3. Process flow of EP (left) and calibrated energy modelling framework (right) 
There are few phases involved in EP technique to obtain the optimum energy baseline configuration.

a) Initialization Phase: The initialization phase is where an initial population of independent variables would be generated within the parameter's corresponding bounds. In this paper, there are three variables that randomized which are the operating hours of axial fan, exhaust fan and water pump that are affecting energy consumption in the building. Constraints of each individual variable are set during this phase to obtain the desired output. The command used to generate random number and constrained are in Equations (1) and (2):

$$
\begin{aligned}
& X i=\operatorname{rand}(K, L) A+B \\
& f \min \leq f i<\max
\end{aligned}
$$

where $\mathrm{K}$ is the number of row, $\mathrm{L}$ is the number of column, $\mathrm{A}$ is the offset, $\mathrm{B}$ is the minimum random, fi is the simulated energy generated from random configuration, $f$ max is the monitored energy in the building, and $f$ min is the minimum acceptable simulated energy. The initialization phase was pre-set to run 1,000 loops or until 20 initial configurations "parents" satisfied the defined constrained.

b) Mutation and Evaluation Phase: The mutation phase is to generate mutated population "offspring" from the parent's population. There are varieties of mutation operator for EP, however this study will use Gaussian mutation as in classic EP. The Gaussian formula is as in Equation (3).

$$
X_{i+m, j}=X_{i, j}+N\left[0, \beta\left(X_{j \max }-X_{j \min }\right)\left(\frac{f_{i}}{f_{\max }}\right)\right.
$$

where, $\mathrm{Xi}+\mathrm{m}, \mathrm{j}$ is the offspring, $\mathrm{Xi}, \mathrm{j}$ is parents, $\beta$ is search step, $\mathrm{Xjmax}$ is maximum parents, $\mathrm{Xjmin}$ is the minimum parents, fi is fitness matrix and fmax is the maximum fitness. In this phase 20 mutated configuration will be generate. The fitness values or also the objective function set in the algorithm is to minimise the error of energy in the system which evaluated using NMBE and CV(RMSE) as shown in Equation (5) and (6).

c) Combination, Selection and New Generation Phase: When new mutated offspring generated, the parents and the offsprings are combined in series to form a group of 40 populations. The population will then sort and rank according to their fitness value in descending order. The top 20 of the population, are then selected and carried forward to form new parents' generation.

d) Convergence Test Phase: Convergence test is to determine the stopping criterion of the simulation. If the difference between the maximum fitness and minimum fitness is zero, the solution is said to be converged and the simulation will stop. The value of accuracy was set to 0.0001 as shown in the Equation (4):

$$
f_{\max }-f_{\min }<0.0001
$$

where $f$ max is maximum energy generate from new parent's population and $f$ min is the minimum energy generate from the same population. If the convergence test fail, the new parent's population will repeat the same process beginning at mutation phase until it's converge.

\subsection{Building Energy Simulator: Energy Plus}

To perform the building simulation, a building model file in IDF format is prepared with weather file for site location. An IDF file consists of a building model data such as building parameters, HVAC setup, electrical equipment etc. IDF file also contains a setting for simulation period and time step which is crucial for communication between simulators. There are two ways to generate IDF file, which is by manually create a new file or modified an older IDF file or by using a 3rd party GUI software. By using the 3rd party software such as Open-studio Energy-plus, users not only able to model the building in 3D but also can adjust the IDF file easily using GUI. Meanwhile, weather data file for the region can be acquired through nearest weather data centre or by downloading from Energy-plus website.

In this paper, Energy-plus runs the simulation using a given energy configuration for a period set by users. The simulation is set for an hourly data acquisition by setting up time step in IDF file. The 'fitness' data generated from building simulation is stored in a database. 


\subsection{Automated Calibration Process}

In order to perform automated calibration for a building energy model, an open source software framework BCVTB developed by the Lawrence Berkeley National Laboratory at the University of California [10] is used as a coupling software. BCVTB allows users to couple different simulator and/or with physical hardware itself for co-simulation. BCVTB employs data exchange mechanism with fixed length of communication interval following it client/server structure. By using Berkeley Software Distribution (BSD) socket interface, inter-process communication allows the actual data exchange take place between simulator clients and BCVTB-server.

In this paper, a co-simulation is built as a prototype framework by coupling two sub-models with each own simulation environment. To build this prototype framework, EnergyPlus and algorithmic optimization engine, Matlab is coupled together to perform optimization where algorithmic and production schedule is integrated in Matlab environment, while, building model is implemented in EnergyPlus. In term of co-simulation, both simulators are run in parallel and exchange their respective input/output data periodically with fixed communication points in hourly manner. EnergyPlus runs the simulation using a given energy configuration for a period set by users. The simulation is set for an hourly data acquisition by setting up time step in IDF file. An IDF file consists of a building model data such as building parameters, HVAC setup, electrical equipment and etc. IDF file also contains a setting for simulation period and time step which is crucial for communication between simulators. The 'fitness' data generated from building simulation is stored in a database.

EP algorithm is written and coded in Matlab environment by sub-dividing into respective phases. The process begins in initialization as to collect 20 initial populations "parents" and ends with convergence test as a function to minimize error. The EP optimization algorithm is used to generate a vectors of random decision configuration and then through BCVTB, coupling framework transfers the data to BES EnergyPlus. From then, EnergyPlus simulates building models with given configuration and test it for a period of time and report the response energy consumption in hourly manner. The process will stop at the end of the loop or until its meet termination criteria. Figure 3 above (right) shows the overall flowchart for the calibration process.

\subsection{Building Energy Evaluation}

Two evaluations from IPMVP, Normalized Mean Biased Error (NMBE) and Coefficient of Variants of Root Mean Squared Error (CV(RMSE)) are used to evaluate the accuracy of the building energy model. The baseline energy is considered optimally calibrated when NMBE and CVRMSE are within acceptable range. Table 1 shows the acceptable MBE and CV(RMSE) tolerance by the IPMVP. NMBE and CV(RMSE) Equations are provided as in (5) and (6) respectively. The optimized energy consumption with the right variables configuration is called calibrated baseline model.

TABLE 1

Acceptable NMBE and CV(RMSE) Tolerance

\begin{tabular}{ccc}
\hline Reporting Type & Index & $\begin{array}{c}\text { Acceptable } \\
\text { Value }\end{array}$ \\
\hline Mothly & NMBE & $\pm 5 \%$ \\
& CV(RMSE) & $15 \%$ \\
\hline Hourly & NMBE & $\pm 10 \%$ \\
& CV(RMSE) & $30 \%$ \\
\hline
\end{tabular}

$$
\begin{gathered}
N M B E=\frac{\sum_{t=1}^{n}\left(y_{i}-\hat{y}_{i}\right)}{(n-p) \hat{y}} \\
C V(R M S E)=\frac{1}{\hat{y}} \sqrt{\frac{\sum_{t=1}^{n}\left(y_{i}-\hat{y}_{i}\right)^{2}}{n-p}}
\end{gathered}
$$

\section{RESULTS AND ANALYSIS}

In this section, the results of the proposed energy model auto calibration approach using tomato greenhouse as the case study is presented. A single objective function which is to minimize error in hourly energy consumption is used in the EP algorithm. The main purposes of this study is to search for the best variables configuration for calibrated energy model that leads to acceptable NMBE and CV(RSME) by IPMVP.

Figure 4 presents the actual daily energy patern that obtained from the greenhouse and used for calibration to compare with the simulation data. The difference between the actual and simulated energy is evaluated in the form of NMBE and CV(RMSE). Figure 5 shows the actual usage of the equipment per hour 
for two weeks. Axial Fan and Exhaust Fan have similar percentage of operating hours which is $44.44 \%$, followed by water pump which is $11.11 \%$.

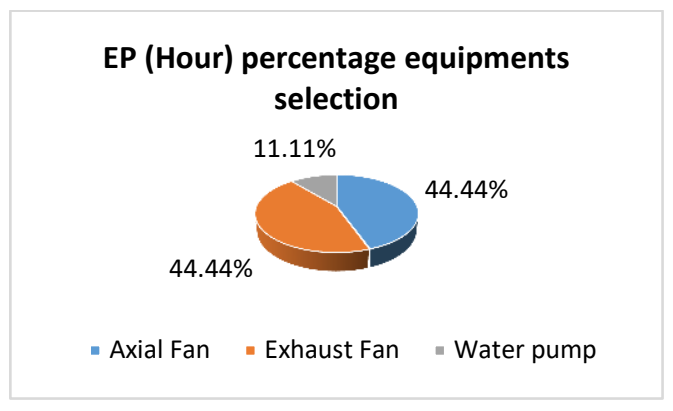

Figure 4. EP (hour) percentage equipment selection

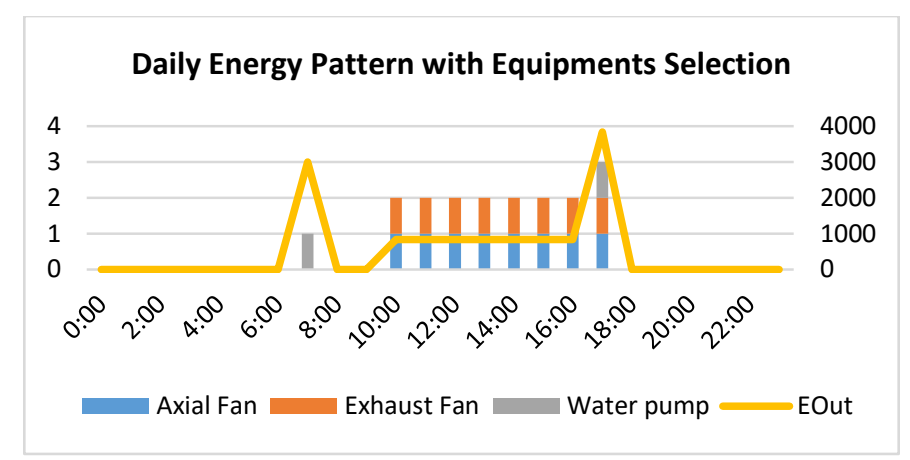

Figure 5. Daily Energy Pattern graph before generating the NMBE and CV(RMSE)

Figure 6 shows the optimal model configuration by EP for Exhaust Fan, Axial Fan and Water Pump in one day. With no weighting factor, the variables configuration is randomized and tested to find optimum calibrated model. As seen in the Figure, the constrained in the model have resulted in operating hour beginning at $6.00 \mathrm{a} . \mathrm{m}$ and closed at $18.00 \mathrm{p} . \mathrm{m}$.

To verify the accuracy of the building energy model, the value of NMBE and CV(RMSE) are calculated. Table 2 shows the NMBE and CV(RMSE) from the automated calibration approach. It is found that the automated approached results in NMBE of $0.01 \%$ and CV(RMSE) of $0.02 \%$. This shows that the accuracy of the building energy model obtained from this simulation is adequate as recommended by IPMVP protocol which requires a tolerance of $\pm 10 \%$ for NMBE and less than $30 \%$ for CV(RMSE) for hourly energy calibration.

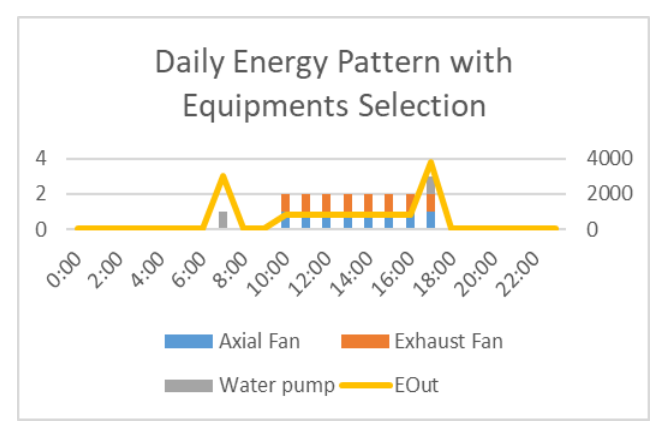

Figure 6. Daily energy pattern with equipment's selection 
Table 2. The NMBE and CV(RMSE) for Simulation-Based Approach

\begin{tabular}{lcc}
\hline Methods & NMBE & CV(RMSE) \\
\hline $\begin{array}{l}\text { Simulation-based } \\
\text { approach }\end{array}$ & 0.01 & 0.02 \\
\hline
\end{tabular}

\section{CONCLUSION}

This study introduces an efficient automated calibration approach to find optimal calibrated energy model for a greenhouse system with minimal error. In this paper, an algorithmic optimization engine, Matlab was coupled with building energy simulator, EnergyPlus using BCVTB as the coupling tool. An Evolutionary Programming (EP) algorithm was chosen and programmed in Matlab to find the best configurations for optimum calibration of greenhouse energy model. Three variables were chosen to find the best configuration which are the operating hours of Exhaust Fan, Axial Fan and Water Pump. The generated variable configuration was sent to EP through BCVTB framework.

The EP generates random variables subjects to a set of pre-defined constraints in the input. The process runs automatically until the EP is converged. The proposed method is not only requiring less computation time but also effective in searching for the best variables configuration with minimal error.

To evaluate the accuracy of the model, NMBE and CV(RMSE) as recommended by the IPMVP were calculated based on the hourly reporting criteria. It is found that the automated calibration approach provides result that is within the recommended standard.

\section{ACKNOWLEDGEMENTS}

We would like to thank Faculty of Electrical Engineering, Universiti Teknologi MARA (UiTM) Shah Alam who has sponsored this paper.

\section{REFERENCES}

[1] IBPSA-USA. Building Energy Software Tools. [Online]. Available: http://www.buildingenergysoftwaretools.com. [Accessed: 21-Nov-2017].

[2] Willy Bernal, Madhur Behl, Truong X. Nghiem, and Rahul Mangharam. (2012). MLE+: A Tool for Integrated Design and Deployment of Energy Efficient Building Controls. 4th ACM Workshop on Embedded Sensing Systems For Energy-Efficiency In Buildings, (BuildSys '12), Toronto, Canada.

[3] University of Pennsylvania, "MLE+: Matlab EnergyPlus Co-Simulation Toolbox." [Online]. Available: mlab.seas.upenn.edu. [Accessed: 21-Nov-2017]

[4] N. Delgarm, B. Sajadi, S. Delgarm, and F. Kowsary. A novel approach for the simulation-based optimization of the buildings energy consumption using NSGA-II : Case study in Iran. Energy Build. 2016Vol: 127, Pages: 552-560.

[5] Valentina Monetti, Elisabeth Davin, Enrico Fabrizio, PhilippeAndre and Marco Filippi. Calibration of building energy simulation models based on optimization- a case study. Energy Procedia. 2015. Issue: 78, pages: 2971-2976.

[6] Haghighat, Laurent Magnier and Fariborz. Multiobjective Optimization of Building Design using TRNSYS Simulations, Genetic Algorithm, and Artificial Neural Network. Building and Environment. 2010. Vol: 45, pages: 739-746,

[7] Navid Delgarm, Behrang Sajadi and Saeed Delgarm. Multi-Objective Optimization of Building Energy Performance and Indoor Thermal Comfort A New Method Using Artificial Bee Colony (ABC). Energy and Buildings. 2016. Issue: 131, pages: 42-53.

[8] T. Hong, J. Kim, J. Jeong, M. Lee, and C. Ji. Automatic calibration model of a building energy simulation using optimization algorithm. Energy Procedia. 2017. Vol: 105, pages: 3698-3704.

[9] T. Yang, Y. Pan, J. Mao, Y. Wang, and Z. Huang. An automated optimization method for calibrating building energy simulation models with measured data: Orientation and a case study. Appl. Energy. 2016. Vol: 179, pages: $1220-1231$.

[10] Wetter, M. Co-Simulation of Building Energy and Control Systems with the Building Controls Virtual Test Bed. Journal of Building Performance Simulation. 2011. Vol: 4(3), pages: 185-203. 